\section{$\underset{\substack{\text { hommes } \\ \text { \& migrations }}}{ }$}

\section{Hommes \& migrations}

Revue française de référence sur les dynamiques

migratoires

$1309 \mid 2015$

Le 3 e âge des migrants

\title{
Les migrants face aux défis du vieillissement
}

\section{Mohamed Madoui et Marcel Jaeger}

\section{OpenEdition \\ Journals}

\section{Édition électronique}

URL : http://journals.openedition.org/hommesmigrations/3060

DOI : 10.4000/hommesmigrations.3060

ISSN : 2262-3353

\section{Éditeur}

Musée national de l'histoire de l'immigration

\section{Édition imprimée}

Date de publication : 1 janvier 2015

Pagination : 6-7

ISBN : 978-2-919040-30-8

ISSN : $1142-852 X$

\section{Référence électronique}

Mohamed Madoui et Marcel Jaeger, «Les migrants face aux défis du vieillissement », Hommes \& migrations [En ligne], 1309 | 2015, mis en ligne le 16 juillet 2015, consulté le 19 avril 2019. URL : http:// journals.openedition.org/hommesmigrations/3060 ; DOI : 10.4000/hommesmigrations.3060 


\section{LES MIGRANTS FACE AUX DÉFIS DU VIEILLISSEMENT}

Par MOHAMED MADOUI, professeur des universités au Cnam, et MARCEL JAEGER, professeur des universités au Cnam, LISE-CNRS.

Réduit à une force de travail au service des activités économiques les plus ingrates et les moins qualifiées, l'immigré n'existe que par, pour et dans le travail, au point que le chômage ou le moment de la retraite finissent par remettre en question la légitimité même de sa présence en terre d'immigration ${ }^{1}$. Appréhendée sous l'unique statut du travail, son existence hors usine n'a été étudiée que récemment. Abdelmalek Sayad a rendu célèbre la formule selon laquelle "les immigrés n'existent qu'en tant qu'ils posent problème" à la société d'accueil. Leur vieillissement suit le même raisonnement : il aura fallu attendre qu'il pose problème pour qu'il devienne, par la force des choses, l'objet des politiques publiques et des investigations académiques.

Sur un plan statistique, de 1990 à 1999, les données issues de l'Institut national de la statistique et des études (Insee) font apparaître une diminution de $22 \%$ de la part des moins de 20 ans dans la population immigrée, tandis que celle des 60 ans et plus a connu une augmentation sensible de $15 \%^{2}$.
Toutes les études prospectives sur la pyramide des âges font le constat d'un vieillissement qui ira en s'accentuant dans les prochaines années. L'invisibilité des migrants vieillissants dans des institutions comme les maisons de retraite ou dans le parc locatif social ou privé se voit compensée par leur surreprésentation dans les foyers de travailleurs migrants. Ces derniers comptent 77198 migrants vivant seuls, auxquels s'ajoutent 44560 personnes résidant dans des parcs diffus (logements meublés, chambres d'hôtel, habitations de fortune, chambres indépendantes, etc.) ${ }^{3}$. Si l'on additionne les immigrés vivant dans des foyers à ceux habitant dans des parcs diffus, on obtient $40 \%$ d'hommes seuls situés hors du parc locatif classique. Ils sont localisés pour la grande majorité d'entre eux dans trois régions françaises : lîle-de-France, ProvenceAlpes-Côte d'Azur et Rhône-Alpes, dans une moindre mesure, la Lorraine et l'Alsace.

Allant à contre-courant de l'idée d'une "vieillesse illégitime" et des discours catastrophistes à l'égard des vieux migrants, Claudine Attias-Donfut 
et Philippe Teissier considèrent au contraire la retraite comme un facteur d'intégration ${ }^{4}$. En s'appuyant sur une enquête réalisée en collaboration entre la Caisse nationale d'assurance vieillesse (Cnav) et l'Institut national de la statistique et des études économiques (Insee) portant sur le vieillissement et le passage à la retraite des immigrés en France (Enquête PRI, Cnav, 2003), ils font le constat que les immigrés trouvent globalement plus d'avantages que d'inconvénients à la retraite. Ils sont également peu nombreux à envisager de rentrer définitivement dans leur pays d'origine $(1,7 \%)$ et une très grande majorité d'entre eux (70 \%) ne ressentent à son égard aucune nostalgie. Ces chiffres sur le non-retour au pays d'origine étonnent un peu et ne vont pas dans le sens des estimations du Haut Conseil à l'intégration (HCI) qui souligne, dans son rapport de 2002, que plus de 90000 personnes ont quitté la France dans les dix dernières années pour s'établir et toucher leur retraite en Algérie, soit un flux de retour d'environ 9100 personnes par an, sans doute le plus important comparativement aux Marocains (1 400 départs/an), aux Espagnols (800 départs/an) et aux Portugais (3 800 départs/an).

À l'heure où se discute au Parlement le projet de loi "Adaptation de la société au vieillissement", ce dossier est l'occasion de décrypter le vieillissement des immigrés sous l'angle des liens familiaux et de la circulation entre la France et les pays d'origine. Il propose de réfléchir plus largement tant sur la place des personnes âgées migrantes dans notre société que sur les dispositifs publics de prise en charge et d'accès aux droits, au logement et aux soins. Il rassemble un ensemble de contributions que l'on répartit en quatre axes principaux.

Le premier axe de ce dossier aborde la situation et les conditions de vie des personnes âgées migrantes en France et en Europe. Laureline Coulomb traite de la question des personnes vieillissantes originaires de l'Union européenne vivant dans la rue. S'appuyant sur une enquête ethnographique, plus particulièrement auprès des migrants européens

vivant dans les rues de Strasbourg, elle décrit les stratégies de survie de ces personnes et les difficultés identitaires qu'elles rencontrent. Dans la même veine, Giulia Fassio analyse dans une perspective historico-anthropologique les conditions de vie et les formes d'autoperception et d'autoreprésentation des personnes âgées d'origine italienne dans la ville de Grenoble. Claudio Bolzman et Laure Kaiser abordent la question des inégalités dans la vieillesse et la santé entre Suisses et diverses catégories de nationalités et de naturalisés, à partir d'une enquête quantitative à questionnaires fermés. À partir d'une enquête empirique récente sous forme de récits de vie qui laisse une large part à la parole des femmes immigrées âgées, Irène Morra et Catherine Gucher analysent les représentations de la santé et de la maladie des femmes immigrées âgées italiennes qui résident en milieu urbain et qui font l'expérience d'une fragilité augmentée par la maladie. Estelle d'Halluin s'interroge, quant à elle, sur les difficultés d'accès aux soins spécifiques à la condition migrante : celles propres aux immigrés enracinés comme celles d'une population moins connue, les

À l'heure où se discute au Parlement le projet de loi "Adaptation de la société au vieillissement", ce dossier est l'occasion de décrypter le vieillissement des immigrés sous l'angle des liens familiaux et de la circulation entre la France et les pays d'origine. primo-arrivants. Enfin, Nawal Bensaid, Jacques Wels et Florence Legros étudient, dans une perspective comparative, les enjeux du vieillissement des populations migrantes sous l'angle de la transportabilité des retraites, des soins de santé et des minima sociaux dans le contexte des accords bilatéraux de Sécurité sociale. Le deuxième axe traite de la question de la maladie d'Alzheimer et de l'accompagnement des personnes âgées migrantes. Jenny Duchier et Jean Mantovani se proposent d'analyser en quoi les répercussions de la maladie d'Alzheimer sur les malades et leur entourage "informel" induisent un ensemble de difficultés dans les familles issues 
de l'immigration maghrébine. Cumulant de nombreux problèmes sociaux (pauvreté, exclusion, vieillesse...), ces familles apparaissent en déficit d'accès à la citoyenneté, aux services et aux soins. Reposant sur l'analyse de 130 articles ou résumés d'articles recensés au cours des cinq dernières années dans la Revue de presse nationale et internationale de la Fondation Médéric Alzheimer entre janvier 2008 et décembre 2013, l'article de Laëtitia Ngatcha-Ribert montre que la maladie d'Alzheimer n'est pas appréhendée de la même façon selon les pays et les régions dans le monde, la communauté, les individus. En réponse à un appel à projets et s'appuyant sur une enquête conduite dans six régions de France auprès d'étudiants et d'intervenants pluridisciplinaires et plurisectoriels, Valérie Wolff et Emmanuel Jovelin cherchent à comprendre les dynamiques à l'œuvre dans l'accompagnement des personnes âgées migrantes. Le troisième axe rassemble des textes variés. C'est le cas de l'article d'Alistair Hunter sur la dépendance familiale aux transferts d'argent et le dilemme du retour au pays d'origine à un âge avancé. L'intérêt de cette contribution est qu'elle aborde la question du vieillissement sous l'angle du genre en comparant les Subsahariens et les Nord-Africains. Mathilde Plard, Aurélien Martineau et Sébastien Fleuret rendent compte de l'avancée des connaissances relatives au processus et aux expériences de vieillissement des personnes âgées immigrées en France depuis les années 1970. En exploitant une revue bibliographique, les auteurs ont saisi les expériences de vieillissement et les ont explorées à travers deux thèmes essentiels : la santé et les conditions d'habitat.

Enfin, le quatrième axe pose la question de la mort et des lieux de sépulture. Au travers de nombreux entretiens menés au cours de plusieurs recherches auprès d'immigrés vieillissants, l'article de Jacques Barou met en évidence la diversité des représentations que les immigrés se font de la mort et leurs souhaits en matière de rites funéraires. S'appuyant sur une enquête qualitative (64 entretiens), Fatima
Mezouj aborde les raisons qui poussent les immigrés à choisir d'être enterrés dans leur pays d'origine et le rapport à la mort des personnes interrogées. Anne-Marie Duguet traite, quant à elle, des attentes en matière de soins (pudeur et propreté), des rites (religieux) et des questions liées à l'inhumation des corps. 